\title{
PERFORMANCE EVALUATION OF MOBILE IP ON MOBILE AD HOC NETWORKS USING NS2
}

\author{
Kingsley K. Ofosu ${ }^{1}$, Jamal-Deen Abdulai $(\mathrm{PhD})^{2}$ and \\ Ferdinand A. Katsriku $(\mathrm{PhD})^{3}$ \\ Department of Computer Science, University of Ghana, Legon-Accra, Ghana
}

\begin{abstract}
Mobile computing devices equipped with transceivers form Mobile Ad Hoc Networks (MANET) when two or more of these devices find themselves within transmission range. MANETs are stand-alone (no existing infrastructure needed), autonomous networks that utilise multi-hop communication to reach nodes out of transmitter range. Unlike infrastructure networks e.g. the Internet with fixed topology, MANETs are dynamic. Despite the heterogeneous nature of these two networks, integrating MANETs with the Internet extends the network coverage and adds to the application domain of MANETs. One of the many ways of combining MANETs with the Internet involves using Mobile Internet Protocol (Mobile IP) and a MANET protocol to route packets between the Internet and the MANET via Gateway agents. In this paper, we evaluate the performance of Mobile IP on MANET in Network Simulator 2 (NS2). We have implemented Mobile IP on Ad hoc On-demand Distance Vector (AODV), Ad hoc On-demand Multiple Distance Vector (AOMDV) and Destination-Sequenced Distance Vector (DSDV) routing protocols and compared performances based on Throughput, End-to-End Delay (E2ED), Packet Delivery Ratio (PDR) and Normalized Packet Ratio (NPR). The simulation results suggest that on-demand routing within the MANET better serves Mobile IP on MANETs.
\end{abstract}

\section{KEYWORDS}

Mobile Ad Hoc Network, Mobile IP, AODV, AOMDV \&DSDV

\section{INTRODUCTION}

Communication has shifted from a predominantly wired setup towards an entirely wireless setup or a merger. Computer networks aid in faster and reliable communications over long distances. The Internet, a network of networks, has become a vital utility in our lives that enable us to communicate around the globe. Mobility as a feature in communication has gained the acceptance of end-users, and so, it is not surprising that Mobile Ad hoc Networks (MANETs) have attracted much attention from researchers. A MANET is an autonomous, infrastructure-less, self-forming and self-repairing data network of mobile devices that support multi-hop communication. MANET could be used to provide Internet connectivity beyond the reach of fixed or cellular infrastructure [1].

The Mobile Internet Protocol (MIP), which was designed to allow mobile nodes to move inside the fixed Internet without losing connectivity, has been experimented in MANET to provide Internet connectivity. We have implemented three variants of Mobile IP on MANET:

Natarajan Meghanathan et al. (Eds) : CCSEA, NCOM, AIFU, DKMP, EMSA, SIPRO, SEA - 2018

pp. 15-27, 2018. (C) CS \& IT-CSCP 2018

DOI : $10.5121 /$ csit.2018.80302 
- Mobile IP on Ad Hoc On-demand Distance Vector (AODV)

- Mobile IP on Ad Hoc On-demand Multiple Distance Vector (AOMDV)

- Mobile IP on Destination-Sequenced Distance Vector (DSDV)

and evaluated their performances using Network Simulator 2 (NS2).

\section{LITERATURE REVIEW}

Not much work has been published concerning providing Internet connectivity for MANETs. A number of publications have suggested corporation between Mobile IP [2] and an ad hoc routing protocol to provide Internet access for MANETs. In "Ad Hoc Networking with Mobile IP" [3], a solution was presented whereby a proactive MANET routing protocol was used with Mobile IP. This solution was not compatible with on-demand routing in MANET. In "MIPMANET - Mobile IP for Mobile Ad Hoc Networks" [4], an on-demand MANET routing protocol (AODV) is used alongside Mobile IP with foreign agent care-of-addresses to connect a MANET to the Internet. Likewise MIPMANET, the Internet draft "Global Connectivity for IPv4 Mobile Ad hoc Networks" (Global4) [5] presented a solution whereby AODV interacts with Mobile IP however, the foreign agent discovery mechanism is incorporated into AODV.

Other publications have used different gateway discovery methods within the MANET routing protocol instead of Mobile IP. The publication titled "Wireless Multihop Internet Access: Gateway Discovery, Routing and Addressing" [6] discusses how MANET nodes can discover gateways to the Internet and the issue of routing and addressing in heterogenous environments. The master's thesis "A Study of Internet Connectivity for Mobile Ad Hoc Networks in NS2" [7] implemented the Internet draft "Global Connectivity for IPv6 Mobile Ad hoc Networks" [8] also known as Global6 in NS2. The AODV routing protocol was modified to include three different gateway detection mechanisms that were tested using NS2 simulations.

Regarding papers that focused more on performance analysis, [9] and [10] conducted separate performance evaluation of Mobile IP on proactive and reactive MANET routing respectively.We will return to these papers as we discuss related works to thus thesis in section 2.3 , after we have discussed Mobile IP and Mobile Ad hoc Networks in Sections 2.1 and 2.2 respectively.

\subsection{Mobile IP}

Mobile IP works by allowing the Mobile Node (MN) to maintain two IP addresses; the home address and the Care-of-Address ( $\mathrm{CoA})$ [2]. The home address is static and binds $\mathrm{MN}$ to a known network called the Home Network (HN). This address is used by transport and application layer protocols to maintain connectivity with MN. On the other hand, the CoA is a dynamic address that $\mathrm{MN}$ obtains as it moves outside $\mathrm{HN}$ into an unknown network termed as a Foreign Network (FN). The CoA reflects the physical location of MN outside of HN.

When MN moves from the $\mathrm{HN}$ into FN, it sends the CoA obtained to the Home Agent (HA) on its $\mathrm{HN}$ via a similar agent on FN known as Foreign Agent (FA). This message prompts HA to receive packets destined for $\mathrm{MN}$ and arriving at $\mathrm{HN}$ on behalf of $\mathrm{MN}$ and then tunnels the packets to the CoA. MN repeats this procedure each time MN obtains a new CoA. There are three building blocks of Mobile IP: agent discovery, registration and datagram delivery. 


\subsubsection{Agent Discovery}

This process is an extension of the router advertisement procedure, as specified in the Internet Control Message Protocol (ICMP) router discovery messages in RFC 1256 [11]. Specifically, the only difference is that an agent advertisement has, also, one or more CoAs made available by FA.

[2] explains that an agent advertisement does the following:

- Allows the detection of mobility agents;

- Lists the available CoA;

- Informs $\mathrm{MN}$ of the services offered by FA e.g. alternative encapsulation techniques;

- Aids MN to determine its network address and the status of the link to the Internet;

- Helps MN to determine whether the agent is a Home Agent, Foreign Agent or both, and therefore whether it is inside $\mathrm{HN}$ or FN.

Agents broadcast advertisements periodically, once a second or once every few seconds. However, an MN may solicit for an agent advertisement regardless of this schedule

\subsubsection{Registration}

When $\mathrm{MN}$ has a new CoA, it must inform HA to render its services to MN. This procedure is known as registration [1]. MN sends a registration request containing:

- Current CoA

- How long MN intends to use the CoA i.e. Time-to-Live (TTL)

- Parameters and flags that specify how HA should forward packets

- Special services that MN requests of HA.

When HA receives the registration request, it then authenticates $\mathrm{MN}$ and then decides whether to accept or reject depending on the outcome of the authentication process. HA sends a reply to MN via FA. If HA accepts the request, it maintains in its cache the home address, CoA and the TTL that MN specified in the request. This trio is known as a binding information, and so registration request is sometimes referred to as a binding update. Although the FA remains passive in this process, it maintains a visitors list of each MN that successfully registers with HA through it.

\subsubsection{Datagram Delivery}

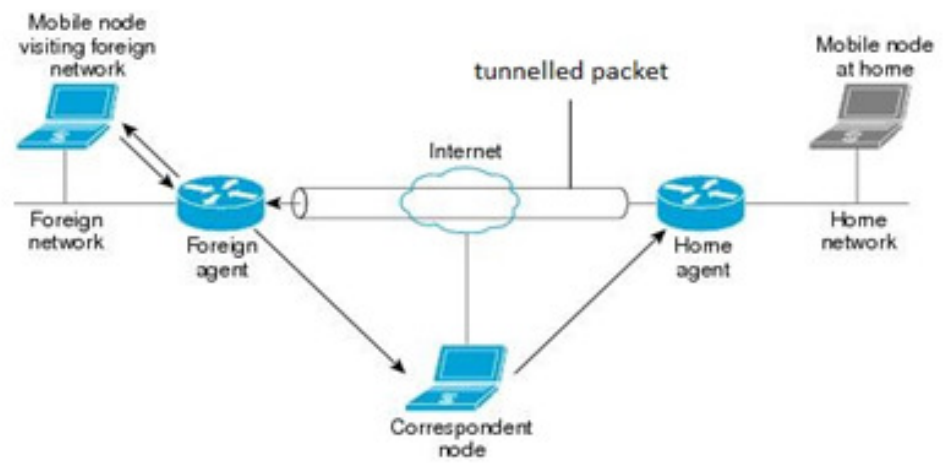

Figure 1. Mobile IP architecture. 
As illustrated in Figure 1 above, the Corresponding Node (CN) transmits packets destined for MN towards HN through standard IP routing. When the packets reach HN, the HA accepts these packets as though it is MN. HA then encapsulates the packet with the CoA as the new destination and forwards the packets to MN. This process is known as tunnelling. Two tunnelling protocols often used are IP within IP [12] and minimal IP within IP [13]. HA continues to receive packets on behalf of MN until TTL runs out. If MN wishes to continue this service, it must register again.

\subsection{Mobile Ad Hoc Networks}

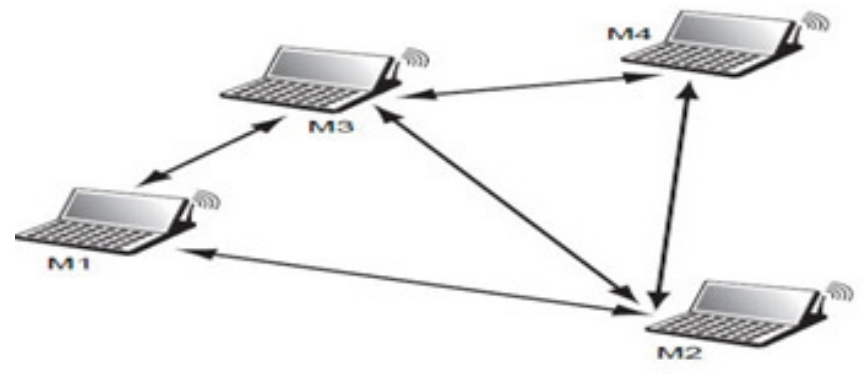

Figure 2. Mobile Ad Hoc Network.

A MANET is an autonomous, infrastructure-less, self-forming and self-repairing network of mobile devices that support multi-hop communication. The mode of transmission is wireless and has a very dynamic topology due to node movements. MANET nodes are usually small, and battery powered. MANETs are deployed in disaster recovery [14], military applications [15], sensor networks [16], etc.

\subsubsection{Manet Routing Protocols}

Further Initially, MANET routing protocols were proactive. They store and update periodically, information on existing paths to all possible destinations for data delivery. Proactive protocols are quicker in choosing routes for data delivery, however, they do not easily converge [17]. E.g., Destination-Sequenced Distance Vector (DSDV) [18].

Reactive or On-demand protocols, unlike the proactive protocols do not anticipate routes between nodes. Nodes discover routes only when there is data to transmit. These protocols only maintain a node neighbour list necessary for route solicitation. E.g. Ad hoc On-demand Distance Vector (AODV) [19].

\section{Destination-Sequenced Distance Vector (DSDV)}

DSDV is based on a distance-vector algorithm and guarantees a loop-free route unlike earlier distance vector based routing protocols by introducing sequence numbers [18]. Nodes maintain a routing table of all destinations with information on the next-hop to the destination, the number of hops and destination sequence number.

Periodically, a node shares its route table information with neighbouring nodes known as route updates. Attached to each update is a sequence number. An odd sequence number indicates an unreachable destination whereas an even sequence number indicates a reachable destination. The greater the sequence number, the fresher the update.

It is possible to have different updates to the same destination with the same sequence number. In such scenarios, nodes prefer the route with a lesser number of hops to the destination. 


\section{Ad Hoc On-Demand Distance Vector (AODV)}

AODV [19] is a reactive protocol also based on the distance-vector algorithm. In AODV, nodes only seek for route information only when they have data to transmit. Loop-free routes are guaranteed with the use of sequence numbers. A node maintains route information only when transmitting data or it finds itself along an active path.

Each time a node has data to share, it solicits for a route to the destination by broadcasting a route request (RREQ) message. A node which is the destination itself or another node that happens to know a route to the destination may unicast a route reply (RREP) to the source or to the intermediate node from which it received the forwarded RREQ and maintains a pointer to that node. This process is known as a forward route setup.

Otherwise, the intermediate node forwards the RREQ and maintains a pointer to the node from which it received the RREQ. This process is known as a reverse route setup. Data transmission may begin as soon as the source receives the RREP via the forward route setup to the destination. Nodes maintain the forward route until there is no more data to transmit or the path becomes broken due to an unreachable intermediate node or destination in which case route solicitation restarts.

\section{Ad Hoc On-Demand Multiple Distance Vector (AOMDV)}

AOMDV [20] is a reactive protocol that improves on AODV. In AOMDV, nodes maintain multiple forward route setups during a single route solicitation procedure. These multiple routes are link disjoint which means that no two routes share a common intermediate node. This procedure reduces the probability that all routes fail at once. A primary route for data transmission is then selected, and nodes maintain the rest as alternate routes. When the primary route fails, nodes select an alternate route for data delivery, unlike AODV that repeats route solicitation whenever there is route failure.

Nodes repeat route solicitation when there is a new transmission session or when all alternate routes and the primary path fails.

\subsection{Related Works}

In this section, we discuss some closely related works to this thesis as we build up the motivation for this study.

In [3], a proposal for connecting MANET to the Internet using Mobile IP is presented in which a modified Routing Information Protocol (RIP) like DSDV was used in routing packets within the MANET. In this proposal, a single routing table is used and shared by Mobile IP and the MANET routing protocol to reduce the management tasks involved in maintaining separate routing tables for Mobile IP and the MANET protocol. Therefore, a route manager is introduced to coordinate route table management between Mobile IP and the modified RIP on the shared routing table. With this, neither Mobile IP or the modified RIP could modify the routing table directly. Route manipulation requests are sent to the route manager which then acts on behalf of the protocols. Although this proposal was successful in providing Internet access to the MANET, it was more proactive and did not support reactive MANET protocols since it relied on the periodic control messages of the MANET routing protocol to propagate agent advertisements.

To fill the gap, [4] presented a master thesis that detailed the use of Mobile IP on a reactive MANET routing protocol. The authors implemented Mobile IP on AODV in NS2 and then 
performed some simulations to validate their study. In this proposal, the authors used Mobile IP with Foreign Agent care-of-addresses to reach Mobile Nodes from the Internet. Packets were exchanged between Home Agent and the Mobile Node via reverse tunnelling to minimize the requirements on AODV. A novel Internetworking unit was introduced between Mobile IP and AODV to ensure that no modifications be made to Mobile IP outside the MANET. The emphasis of the thesis was not on performance evaluation and so the simulations were not quite extensive.

Also, the Internet draft "Global Connectivity for IPv4 Mobile Ad Hoc Networks" [5] sometimes referred to as Global4, presented a solution which involves the use of Mobile IP with foreign agent care-of-addresses and AODV just as was done in MIPMANET. However, foreign agent discovery was made a part of AODV while mobile node registrations with the foreign agent via Mobile IP is maintained.

In the paper titled "Wireless Multihop Internet Access: Gateway Discovery, Routing and Addressing" [6], an alternative approach to providing Internet Access for MANETs is describes. In this approach, Mobile IP is eliminated. The use of specific routers that serve as gateways resolve the heterogeneity between the fixed Internet and the MANET. Although the solution looks promising, it was based on IPv6 networks which has not gained popularity over IPv4 networks.

Again, the master's thesis “A Study of Internet Connectivity for Mobile Ad Hoc Networks in NS2" [7] implemented the Internet draft "Global Connectivity for IPv6 Mobile Ad hoc Networks" [8] also known as Global6 in NS2. Global6 is an enhancement on Global4 however, Mobile IP services are not used. The AODV routing protocol was modified to include three different gateway detection mechanisms that were tested using NS2 simulations. The gateway detection mechanisms were classified as proactive, reactive and hybrid. The results from simulations showed no significant difference in packet delivery ratio between the three gateway discovery mechanisms. However, the proactive and hybrid mechanisms performed slightly better than the reactive mechanism regarding average end-to-end delay.

With regards to measuring performance of Mobile IP on MANET, [9] evaluated the performance of Mobile IP on a proactive MANET protocol, DSDV. They created simulation scenarios involving a Home Agent, three Routers, four Foreign Agents, a Corresponding Node and a varying number of Mobile Nodes at varying speeds. Number of nodes were varied to test the robustness of the solution as traffic increases within the simulation space. This was done to rightly model the real Internet world, as we are witnesses to the increasing number of mobile devices on the Internet. They evaluated performance of Mobile IP based on received packets, lost packets, throughput and End-to-End Delay (E2ED).

Also, [10] performed simulations in NS2 to evaluate the performance of Mobile IP on AODV. They used a single Home Agent and one Foreign Agent on a $670 \times 670$ rectangular field with a few Mobile Nodes. Performance metrics used were throughput, delay and packet overhead. Although the methodology was convincing, the simulation setup was poorly described.

In our thesis, we take these works further as we present on the same simulation platform, scenarios to evaluate the performance of Mobile IP on both reactive and proactive MANET routing on IPv4 networks. Our choice of solution involving corporation between Mobile IP and a MANET routing protocol over methods involving IPv6 is mainly because IPv4 as of now is still widely used over IPv6.

We evaluate the performance of Mobile IP on MANET by performing simulations in Network Simulator 2 (NS2). Likewise in [9], we vary number of nodes in our simulation scenarios to account for increasing traffic within the network. 


\section{METHODOLOGY}

As mentioned earlier, we modelled a Mobile Ad Hoc Network using Network Simulator 2 software. Due to the overwhelming dynamism and cost involved in running a live experiment, we chose a computer simulation model specifically using NS2 which has a widely accepted error margin within the networking research community.

\subsection{Simulation Scenario}

We used a square flat surface of dimension $670 \mathrm{~m} \times 670 \mathrm{~m}$ with a simulation time of $200 \mathrm{sec}$ as seen in Figure 3. We modelled the fixed Internet using a Home Agent and four Foreign Agents all with a transmission range of $100 \mathrm{~m}$. We placed a gap of $5 \mathrm{~m}$ between any two adjacent agents. The gap was to ensure that Mobile Nodes decide between Foreign Agents quickly. We simulate up to 175 Mobile Nodes. For each number of Mobile Nodes selected, we performed at least 10 different simulation runs and averaged our results.

Table 1. Simulation Parameters.

\begin{tabular}{|l|l|}
\hline Parameter & Value \\
\hline Movement model & Random waypoint \\
\hline Traffic type & CBR \\
\hline MAC layer & 802.11 \\
\hline Transmitter range & $\sim 100 \mathrm{~m}$ \\
\hline Bandwidth & $5 \mathrm{Mbps}$ \\
\hline Simulation time & $200 \mathrm{sec}$ \\
\hline Simulation field & $670 \mathrm{~m} \times 670 \mathrm{~m}$ \\
\hline $\begin{array}{l}\text { Number of foreign } \\
\text { agents }\end{array}$ & 4 \\
\hline Packet rate & $1 \mathrm{Mbps}$ \\
\hline Pause time & 0 \\
\hline Maximum speed & $5 \mathrm{~m} / \mathrm{s}$ \\
\hline
\end{tabular}

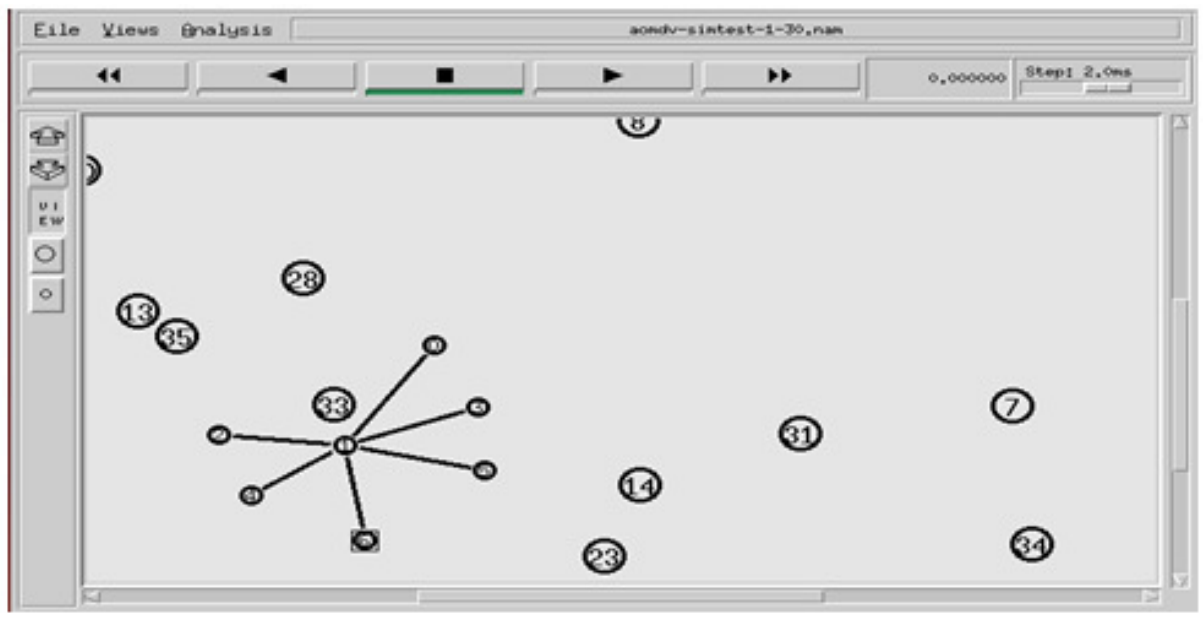

Figure 3. Wired cum wireless scenario. 


\section{RESULTS AND DISCUSSION}

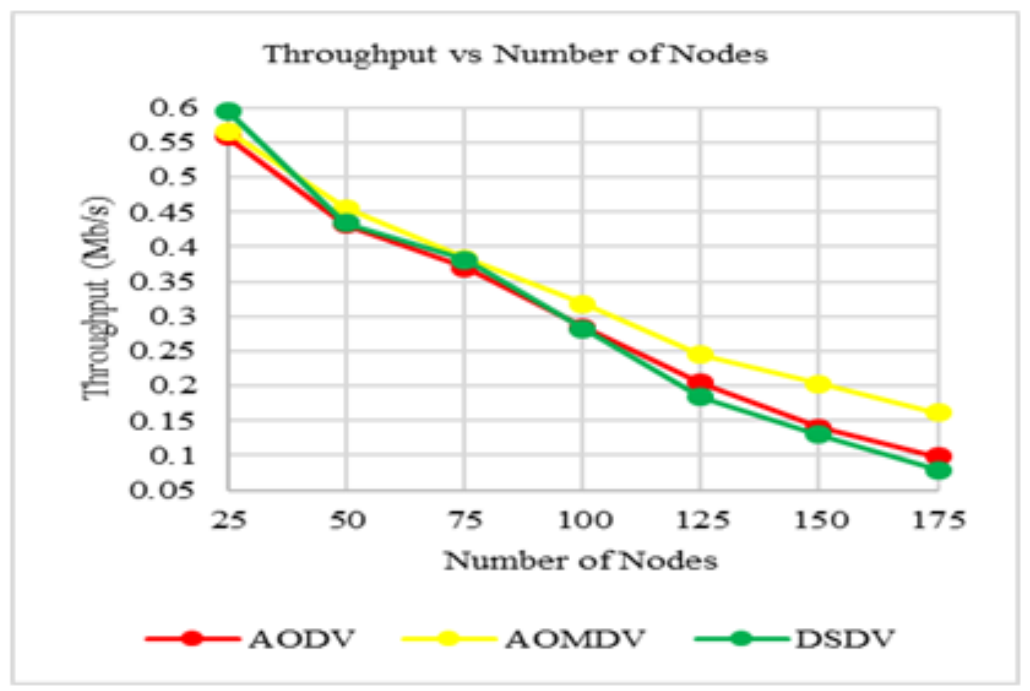

Figure 4. Throughput in $\mathrm{Mb} / \mathrm{s}$.

Figure 4 above shows the network throughput as recorded in our simulations. Throughput is a measure of the actual data that can be sent across a channel per unit time. Throughput is usually lesser than channel bandwidth because, channel bandwidth is just a theoretical estimation of how much data could be sent across a channel per unit time. In measuring the network throughput, we excluded control packets and focused on the CBR data packets only. This is sometimes referred to as Goodput. Our results show that, throughput decreases as number of nodes increase. AOMDV had the best throughput performance whereas DSDV had the worst, as number of nodes increased. The observed difference in performance is because, AOMDV sends fewer control messages to nodes than AODV and DSDV and so the channel is less occupied and data packets can be transmitted with ease.

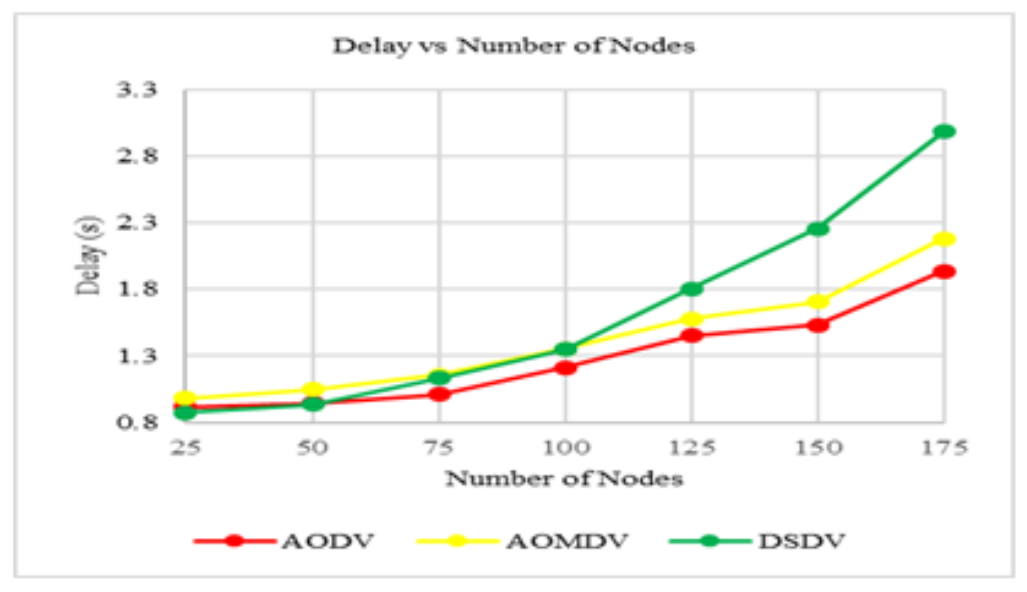

Figure 5. Average end-to-end delay.

Average End-to-End Delay (E2ED) is a measure of how fast a unit size of data can be transmitted across a network. E2ED is a sum of propagation delay, queueing delay, transmission delay and processing delay at intermediate nodes. E2ED is influenced by node mobility and transmission distance. Frequent mobility and longer distances result in longer E2ED. In our simulations, the 
same mobility files were used in all three implementations to nullify the effects of mobility and distances when comparing E2ED performances. In all 3 implementations, E2ED increased as nodes increased. AODV and AOMDV showed similar trends as number of nodes increased. Since they are both On-demand routing protocols, path discovery affects E2ED. AOMDV and AODV use the same route discovery mechanism and deliver packets in a similar fashion therefore, within the same mobility scenario they are expected to have the same E2ED. The extra delay recorded by AOMDV is because it spends additional time during each route discovery process to discover multiple routes. DSDV had the worst E2ED performance with a sharp rise in E2ED trend as number of nodes increased. The observed difference is because, intermediate nodes spend longer processing delays as they check their route tables for next hops to forward data, whereas AOMDV and AODV use forward route setups resulting in less processing delays at intermediate nodes.

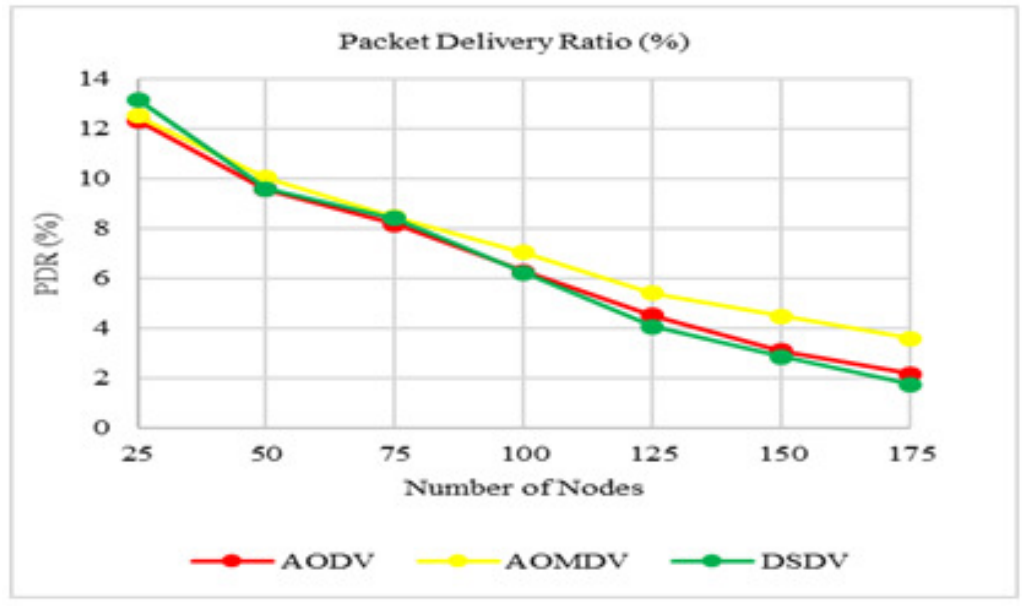

Figure 6. Packet delivery ratio.

Packet Delivery Ratio (PDR) is the ratio of delivered packets to the total number of packets generated. In figure 6 above, we expressed this ratio as a percentage. PDR is inversely correlated to Packet Loss Ratio. A higher packet delivery ratio means fewer packet loss. AOMDV proved to be the most reliable in terms of packet delivery. As mentioned earlier, AOMDV discovers multiple disjoint paths between source and destination. These disjoint paths are less probable to fail all at once hence, a higher reliability in delivering packets from source to destination.

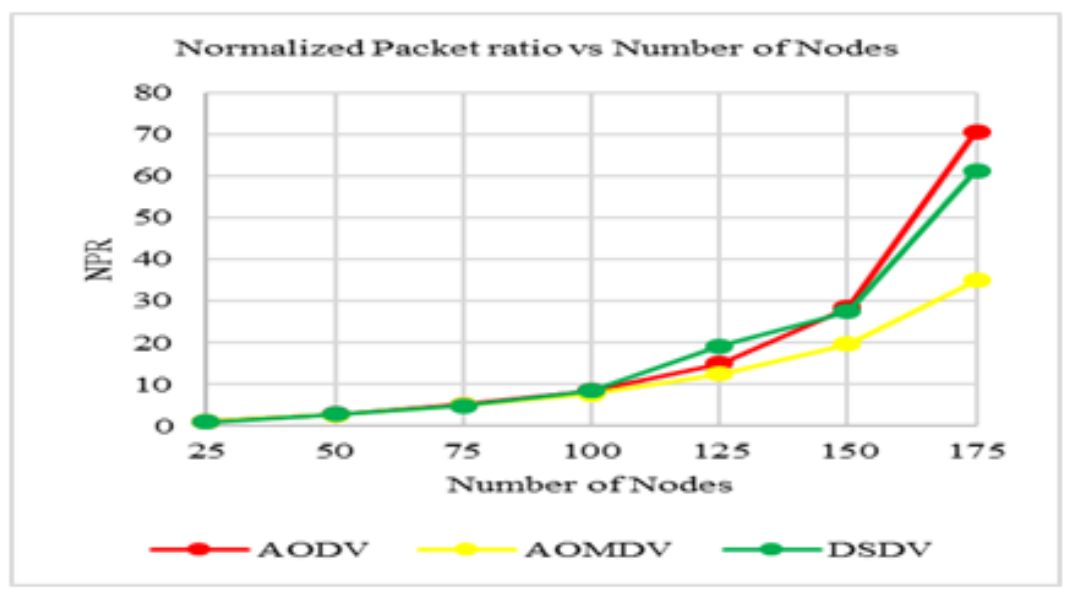

Figure 7. Normalized packet ratio. 
Normalized Packet Ratio (NPR) is the ratio of control packets to actual data packets. NPR can be interpreted as the number of control messages needed by the MANET routing protocol to send a single data packet from source to destination. A lesser NPR is most preferred in networks as control messages are not useful to end users. In figure 7, it is observed that, all three protocols have similar trend and values from 25 to 100 nodes. The significant difference in trend begins after 100 nodes. AOMDV had the best NPR performance whereas AODV recorded the worst performance. The observed pattern is attributed to the fact that AODV nodes repeat the route discovery procedure each time a link is broken unlike AOMDV that resorts to a secondary path earlier discovered during the immediate past route discovery phase. It is only when all the multiple paths fail that AOMDV nodes repeat the route discovery procedure.

In figure 8 and figure 9 below, we zoom in on figure 7 to show a better picture of the trends between 25 and 100 nodes.

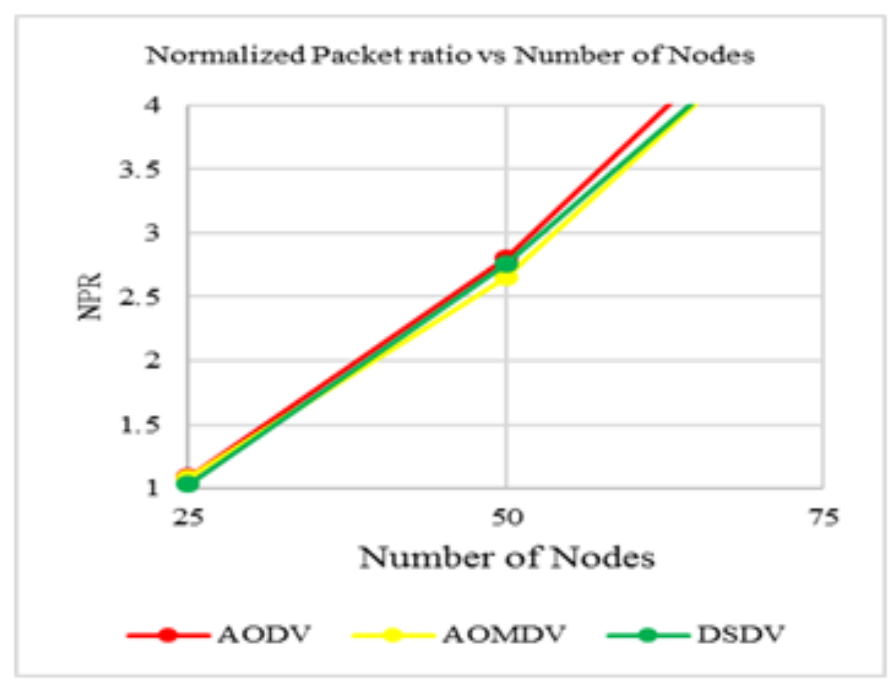

Figure 8. Normalized packet ratio (A).

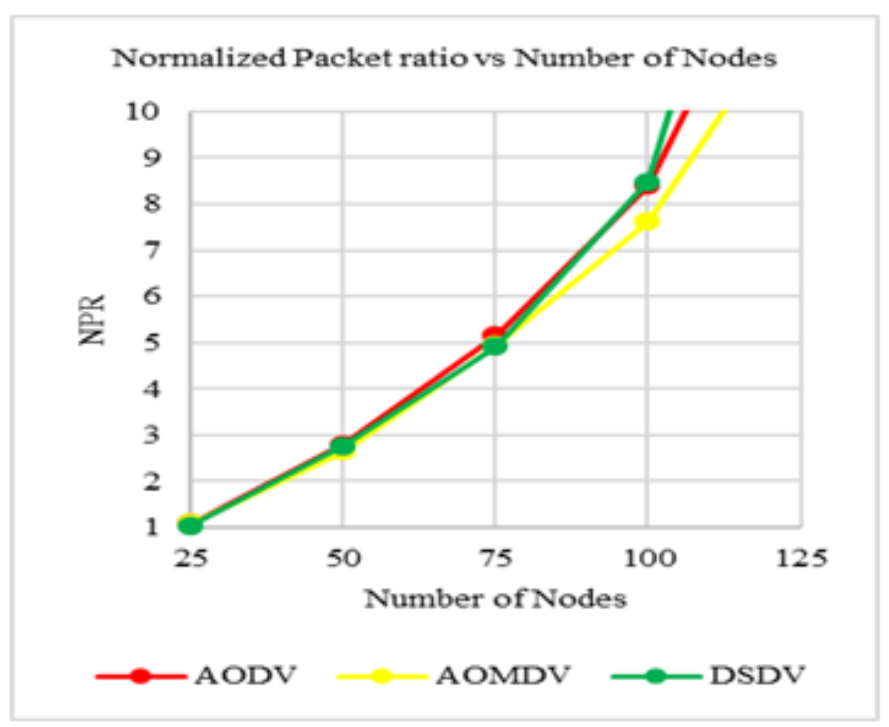

Figure 9. Normalized packet ratio (B). 


\section{CONCLUSIONS}

We have presented a performance evaluation of Mobile IP on MANET. We have studied for the first time the performance of Mobile IP on MANET using Ad Hoc On-demand Multiple Distance Vector (AOMDV) for routing packets inside the MANET. We have also studied the performance of Mobile IP using Destination-Sequenced Distance Vector (DSDV) and Ad Hoc On-demand Distance Vector (AODV) for routing packets inside the MANET.

We modelled Mobile IP on MANET using a wired cum wireless scenario in NS2. Our scenario included one Home Agent, a Corresponding Node and four Foreign Agents. Mobile nodes moved randomly between these sub networks and registered with an agent whenever they entered a new sub network. Communication between Mobile Nodes and Corresponding Node continued even as Mobile Nodes moved across sub networks.

Results obtained from simulation suggested that On-demand routing improved the performance of Mobile IP on MANET regarding the average end-to-end delay, throughput, packet delivery ratio and normalized packet ratio. Specifically, Mobile IP on AOMDV outperformed AODV and DSDV.

\section{ACKNOWLEDGEMENTS}

We are grateful to God for the gift of life and good health throughout this study. We thank family, friends and colleagues for the support and encouragements thus far.

\section{REFERENCES}

[1] U. Jönsson, F. Alriksson, T. Lasson, P. Johansson, and G. Maguire, "MIPMANET - Mobile I P for Mobile Ad Hoc Networks," MobiHoc '00 Proc. 1st ACM Int. Symp. Mob. ad hoc Netw. Comput., pp. 75-85, 2000.

[2] C. E. Perkins, "Mobile networking through mobile IP," IEEE Internet Comput., vol. 2, no. 1, pp. 5869, 1998.

[3] H. Lei and C. Perkins., "Ad Hoc Networking with Mobile IP," in 2nd European Personal Mobile Communications Conf. (EPMCC 97), IEE., 1997, p. 197-202.

[4] U. Jönsson, F. Alriksson, T. Larsson, P. Johansson, G. Maguire, and Jr, "MIPMANET: mobile IP for mobile ad hoc networks,” MobiHoc '00 Proc. 1st ACM Int. Symp. Mob. ad hoc Netw. Comput., pp. $75-85,2000$.

[5] E. M. Belding-Royer, Y. Sun, and C. E. Perkins, "Global Connectivity for IPv4 Mobile Ad hoc Networks, IETF Internet Draft.” Work in progress, 2001.

[6] J. Xi and C. Bettstetter, "WIRELESS MULTIHOP INTERNET ACCESS : GATEWAY DISCOVERY , ROUTING , AND ADDRESSING,” Proc. Int. Conf. Third Gener. Beyond, 2002.

[7] A. A. Hamidian, "A Study of Internet Connectivity for Mobile Ad Hoc Networks in NS 2," no. January, 2003.

[8] R. Wakikawa, J. T. Malinen, C. E. Perkins, A. Nilsson, and A. J. Tuominen, "Global Connectivity for IPv6 Mobile Ad hoc Networks, IETF Internet Draft.” Work in progress, 2001.

[9] E. Hassan, A. Alsaied, S. M. Alshareefmodatheir, and I. Hal, "Performance Evaluation of Mobile IP with DSD V Routing Protocol using NS2," 2015. 
[10] S. N. Mane, N. V. Mane, and D. G. Khairnar, "Performance of mobile node between different MANET with Mobile IP,” 2015 Int. Conf. Ind. Instrum. Control. ICIC 2015, no. Icic, pp. 1662-1664, 2015.

[11] S. Deering and Ed., "ICMP Router Discovery Messages," no. RFC 1256. pp. 1-19, 1991.

[12] C. E. Perkins, "IP Encapsulation within IP,” no. RFC 2003. pp. 1-14, 1996.

[13] C. E. Perkins, "Minimal Encapsulation within IP,” no. RFC 2004. pp. 1-6, 1996.

[14] D. G. Reina et al., "A survey on ad hoc networks for disaster scenarios," in Proceedings - 2014 International Conference on Intelligent Networking and Collaborative Systems, IEEE INCoS 2014, 2014, pp. 433-438.

[15] R. Ruppe, S. Griswald, P. Walsh, and R. Martin, "Near Term Digital Radio (NTDR) System." IEEE, pp. 1282-1287, 1997.

[16] M. M. N. Aldeer, “A summary survey on recent applications of wireless sensor networks," Res. Dev. (SCOReD), 2013 IEEE Student Conf., no. December, pp. 485-490, 2013.

[17] C. E. Perkins, “Ad Hoc Networking,” p. 370, 2000.

[18] C. E. Perkins and P. Bhagwat, "Highly Dynamic ( DSDV ) for Mobile Computers Routing," Proc. ACM SIGCOMM94, London, UK, pp. 234-244, 1994.

[19] C. E. Perkins and E. M. Royer, "Ad-hoc on-demand distance vector routing," Proc. - WMCSA'99 2nd IEEE Work. Mob. Comput. Syst. Appl., pp. 90-100, 1999.

[20] M. K. Marina and S. R. Das, “Ad hoc On-demand Multipath Distance Vector Routing," ACM SIGMOBILE Mob. Comput. Commun. Rev., vol. 6, no. 3, pp. 92-93, 2002.

\section{AUTHORS}

Kingsley K. Ofosu is currently an MPhil student at the Department of Computer Science, University of Ghana. He received his BSc. degree in Psychology and Computer Science (Combined) from University of Ghana in 2013. His research interests include mobile networks, cloud computing and intelligent systems.

Jamal-Deen Abdulai received his BSc. degree in Computer Science in 2002 from the Kwame Nkrumah University of Science and Technology (KNUST). In 2006, he was awarded an MPhil and later in 2009 a PhD in Computer Science by the Department of Computing Science at the University of Glasgow, UK.

Dr. Abdulai's is currently a lecturer at the Department of Computer Science, University of Ghana, investigating how probabilistic methods can be used to optimize the performance of both wired and wireless networks. His research interests include Performance modelling and evaluation of Mobile Wireless Ad hoc and Sensor

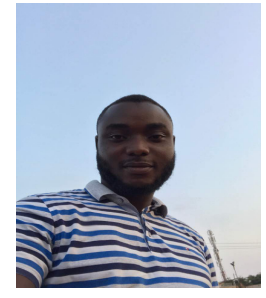
Networks, Network Security and Management, Embedded Systems, Parallel and Distributed Systems, Artificial Intelligence and its application in information security. 
Ferdinand A. Katsriku is the current head of the department of computer science, University of Ghana. In 1989, he received an M. Eng. in computer systems engineering with distinction from Kharkov Polytechnic Institute. He was awarded an MSc in Laser Engineering and Pulsed Power Technology in August 1992 and later awarded postgraduate certificate in education from King's College London in 1996. In 2000, he received a $\mathrm{PhD}$ in Information Engineering from City University, London. His current research interests are wireless sensor networks, cognitive radio and water quality monitoring. He is also a technical Reviewer of EPSRC grants and for major publishers including John Wiley and Elsevier.

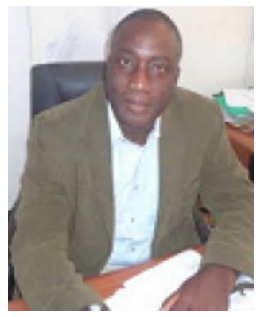

